\title{
Exact solutions of Einstein-æther gravity in Bianchi type $\mathbf{V}$ cosmology
}

\author{
M. Roumeliotis ${ }^{1, \mathrm{a}}$, A. Paliathanasis ${ }^{2,3, \mathrm{~b}}$, Petros A. Terzis ${ }^{1, \mathrm{c}}$, T. Christodoulakis ${ }^{1, \mathrm{~d}}$ (i) \\ ${ }^{1}$ Nuclear and Particle Physics Section, Physics Department, University of Athens, 15771 Athens, Greece \\ ${ }^{2}$ Institute of Systems Science, Durban University of Technology, POB 1334, Durban 4000, South Africa \\ ${ }^{3}$ Instituto de Ciencias Físicas y Matemáticas, Universidad Austral de Chile, 5090000 Valdivia, Chile
}

Received: 10 November 2019 / Accepted: 3 March 2020 / Published online: 13 March 2020

(C) The Author(s) 2020

\begin{abstract}
We present the solution space of the field equations in the Einstein-æther theory for the case of a vacuum Bianchi Type $V$ space-time. We also find that there are portions of the initial parameters space for which no solution is admitted by the reduced equations. Whenever solutions do exist, their physical interpretation is examined through the behavior of Ricci and/or Kretsmann scalar, as well as with the identification of the effective energy momentum tensor in terms of a perfect fluid. There are cases in which no singularities appear and others where the effective fluid is isotropic.
\end{abstract}

\section{Introduction}

Bianchi spacetimes contain various important cosmological models that have been used to describe the anisotropies of primordial universe and the evolution to an isotropic universe as observed in the present time [1-4]. In Bianchi models the spacetime manifold is foliated along the time axis, with three dimensional homogeneous hypersurfaces [5]. The corresponding geometries are thus spatially homogeneous, which means that the physical variables depend on the time variable only, reducing the Einstein and other governing equations to coupled ordinary differential equations. In total there are nine Bianchi models which are defined by the possible nine different three-dimensional algebras constructed from the isometries of the metric tensor (see for example $[5,6])$.

In the context of GR, the field equations for Bianchi spacetimes admit exact solutions, for instance see [7-17] and references therein. In the case where a matter source is included

\footnotetext{
a e-mail: microum@phys.uoa.gr

be-mail: anpaliat@phys.uoa.gr

ce-mail: pterzis@phys.uoa.gr

de-mail: tchris@phys.uoa.gr (corresponding author)
}

in Einstein's GR, such as electromagnetic fluid, ideal gas and scalar field, some exact solutions were previously determined in [18-27].

In this work we are interested in the exact solutions of field equations for Bianchi $\mathrm{V}$ spacetime in Einstein-aether gravity $[28,29]$. This is a Lorentz violating gravitational theory, because a preferred frame is introduced through the aether field present in the gravitational action integral [30]. Mathematically, the aether field is described by a unit time-like vector field, with its kinematic quantities coupled to gravity in the Einstein-Hilbert action. By definition Einstein-æther gravity is a second-order theory however, because of the existence of nonlinear terms in the aether field, there are very few exact solutions. For applications of Einstein-aether gravity in cosmological studies we refer the reader to [31-35].

Recently, in [36] exact solutions were found for the case of Friedmann-Lemaître-Robertson-Walker (FLRW) and LRS Bianchi III spacetimes in Einstein-aether theory. It was there proved that exact solutions exist only for specific ranges of values of the free coupling parameters for the aether field. These exact solutions have been applied in [37] for the determination of inhomogeneous cosmological solutions in Einstein-aether theory. Other studies which can be found in the literature on anisotropic Einstein-aether gravitational models are based on the method of the critical point analysis, where special solutions are determined at the critical points which describe asymptotic behavior for the evolution of the field equations [38-42]. The plan of the paper is as follows:

In Sect. 2 we briefly present the field equations in Einsteinaether gravity. Section 3 includes the main material of our study where we present the solution of the field equations for the case where the aether field is either parallel to the comoving observer (Class A), or tilted (Class B). For these two classes we find the relations between the coupling constants $c_{i}$ of the theory in order that the field equations do admit solutions. For both Classes there are anisotropic solu- 
tions, whereas in the first class, where the aether field is parallel to the comoving observer, there is an isotropic solution which corresponds to a FLRW spacetime with nonzero spatial curvature. Furthermore, the physical description of the corresponding energy momentum tensor is calculated in all cases and is then used to understand the physical properties of the resulting spacetimes. Finally, at Sect. 4 we draw our conclusions.

\section{Einstein-æther gravity}

The action integral in Einstein-æther gravity is

$$
\begin{aligned}
S_{A E}= & \int d^{4} x \sqrt{-g} R+\int d^{4} x \sqrt{-g}\left(K^{\alpha \beta \mu \nu} u_{\mu ; \alpha} u_{v ; \beta}\right. \\
& \left.+\lambda\left(u^{\alpha} u_{\alpha}+1\right)\right),
\end{aligned}
$$

where $u^{\alpha}$ is the aether field i.e. a unit time-like vector field, which defines the preferred frame. The Lagrange multiplier $\lambda$ ensures the unitarity of the aether field, $u^{\alpha} u_{\alpha}=-1$. Finally, the Lorentz violating terms are introduced by the tensor $K^{\alpha \beta \mu \nu}$ defined as

$$
\begin{aligned}
K^{\alpha \beta \mu \nu} \equiv & c_{1} g^{\alpha \beta} g^{\mu \nu}+c_{2} g^{\alpha \mu} g^{\beta \nu} \\
& +c_{3} g^{\alpha \nu} g^{\beta \mu}+c_{4} g^{\mu \nu} u^{\alpha} u^{\beta} .
\end{aligned}
$$

where $c_{1}, c_{2}, c_{3}$ and $c_{4}$ are the dimensionless coupling parameters of the aether field with the gravity.

In [43] the first two terms of action integral (2.1) have been written with the use of the kinematic quantities of the aether field in $1+3$ analysis as follows

$$
\begin{aligned}
S_{E A}= & \int \sqrt{-g} d x^{4}\left(R+c_{\theta} \theta^{2}+c_{\sigma} \sigma^{2}\right. \\
& \left.+c_{\omega} \omega^{2}+c_{\alpha} \alpha^{2}+\lambda\left(u^{\alpha} u_{\alpha}+1\right)\right)
\end{aligned}
$$

where

$$
\begin{aligned}
\alpha_{\mu} & =u_{\mu ; \nu} u^{\nu}, \theta=\frac{1}{3} u_{\mu ; \nu} h^{\mu \nu}, \\
\sigma_{\mu \nu} & =u_{(\alpha ; \beta)} h_{\mu}^{\alpha} h_{\nu}^{\beta}-\theta h_{\mu \nu}, \\
\omega_{\mu \nu} & =u_{[\alpha ; \beta]} h^{\alpha \beta} h_{\mu \nu}, \sigma^{2}=\sigma^{\mu \nu} \sigma_{\mu \nu}, \\
\omega^{2} & =\omega^{\mu \nu} \omega_{\mu \nu},
\end{aligned}
$$

with $\alpha^{\mu}$ the acceleration, $\theta$ the expansion rate, $\sigma_{\mu \nu}$ the shear tensor, $\omega_{\mu \nu}$ the vorticity tensor and $h_{\mu \nu}$ the projective tensor defined as $h_{\mu \nu}=g_{\mu \nu}-u_{\mu} u_{\nu}$. The coefficient constants $c_{\theta}, c_{\sigma}, c_{\omega}$ and $c_{a}$ are related with $c_{1}, c_{2}, c_{3}$ and $c_{4}$ as follows

$$
\begin{aligned}
c_{\theta} & =\frac{1}{3}\left(3 c_{2}+c_{1}+c_{3}\right), \\
c_{\sigma} & =c_{1}+c_{3}, c_{\omega}=c_{1}-c_{3}, c_{a}=c_{4}-c_{1} .
\end{aligned}
$$

The gravitational field equations of the Einstein-aether theory are obtained by variation of (2.1) with respect to the metric $g^{\mu \nu}$, fielding

$G_{\mu \nu}=T_{\mu \nu}^{\mathfrak{x}}$,

where $G_{\mu \nu}$ is the Einstein tensor and $T_{\mu \nu}^{\mathfrak{x}}$ is the effective æther energy-momentum tensor which is defined as

$$
\begin{aligned}
T_{\mu \nu}^{\mathfrak{x}}= & \frac{1}{2} g_{\mu \nu} K^{\alpha \beta \rho \sigma} u_{\rho ; \alpha} u_{\sigma ; \beta}+\frac{1}{2} g_{\mu \nu} \lambda\left(g^{\alpha \beta} u_{\alpha} u_{\beta}+1\right) \\
& -c_{1} g^{\alpha \beta}\left(u_{\alpha ; \mu} u_{\beta ; \nu}+u_{\mu ; \alpha} u_{v ; \beta}\right) \\
& -c_{2} g^{\alpha \beta}\left(u_{\mu ; \nu} u_{\alpha ; \beta}+u_{\beta ; \alpha} u_{v ; \mu}\right) \\
& -c_{3} g^{\alpha \beta}\left(u_{\alpha ; \mu} u_{v ; \beta}+u_{\mu ; \alpha} u_{\beta ; \nu}\right) \\
& -c_{4} u^{\alpha} u^{\beta} u_{\mu ; \alpha} u_{\nu ; \beta} \\
& -c_{4}\left(g^{\lambda \alpha} u_{\mu} u^{\beta} u_{\lambda ; \nu} u_{\alpha ; \beta}+g^{\beta \lambda} u^{\alpha} u_{\nu} u_{\beta ; \alpha} u_{\lambda ; \mu}\right)-\lambda u_{\mu} u_{\nu} \\
& +\left(\frac{1}{2} K^{\alpha \beta \lambda \kappa} u^{\rho} u_{\kappa ; \beta} g_{\rho \mu} g_{\lambda \nu}+\frac{1}{2} K^{\alpha \beta \lambda \kappa} u^{\rho} u_{\kappa ; \beta} g_{\rho \nu} g_{\lambda \mu}\right)_{; \alpha} \\
& +\left(\frac{1}{2} K^{\alpha \beta \lambda \kappa} u^{\rho} u_{\kappa ; \beta} g_{\rho \mu} g_{\alpha \nu}+\frac{1}{2} K^{\alpha \beta \lambda \kappa} u^{\rho} u_{\kappa ; \beta} g_{\rho \nu} g_{\alpha \mu}\right)_{; \lambda} \\
& -\left(\frac{1}{2} K^{\alpha \beta \lambda \kappa} u^{\rho} u_{\kappa ; \beta} g_{\lambda \mu} g_{\alpha \nu}+\frac{1}{2} K^{\alpha \beta \lambda \kappa} u^{\rho} u_{\kappa ; \beta} g_{\lambda \nu} g_{\alpha \mu}\right)_{; \rho} .
\end{aligned}
$$

The equation of motion for the aether field follows by varying $u_{a}$

$$
\begin{aligned}
& c_{4} g^{\mu \nu} u^{\alpha} u_{v ; \beta} u_{\mu ; \alpha} g^{\kappa \beta}-c_{4} g^{\mu \kappa} g^{\alpha \lambda} u_{\lambda ; \beta} u^{\beta} u_{\mu ; \alpha} \\
& \quad-c_{4} g^{\mu \kappa} u^{\alpha} u_{; \beta}^{\beta} u_{\mu ; \alpha}-K^{\alpha \beta \mu \kappa} u_{\mu ; \alpha ; \beta}+\lambda g^{\alpha \kappa} u_{\alpha}=0
\end{aligned}
$$

while its constraining unit condition is obtained by varying $\lambda$

$u^{a} u_{a}+1=0$.

The above equations make it clear that Einstein-æther gravity is a second-order theory; in a four dimensional manifold this system comprises fifteen equations. It is important to mention that in our consideration we have not assumed any matter content and the effectiveæther energy-momentum tensor is produced from the second term of the action (2.1).

\subsection{Physical interpretation}

In order to assign a possible physical meaning to the solutions obtained we calculate, on mass-shell, the Einstein tensor $G_{\mu \nu}=R_{\mu \nu}-\frac{1}{2} R g_{\mu \nu}$ and interpret it as an effective energymomentum tensor of a perfect fluid by writing $G_{\mu \nu}=T_{\mu \nu}^{(e f f)}$. The energy momentum tensor in the $1+3$ decomposition can be written as follows

$T_{\mu \nu}^{(e f f)}=(\rho+p) u_{\mu} u_{\nu}+p g_{\mu \nu}+2 q_{(\mu} u_{\nu)}+\pi_{\mu \nu}$,

where $\rho$ is the energy density of the fluid, $u_{\mu}$ the 4-velosity, $q_{\mu}$ the heat flux vector, $\mathrm{p}$ the pressure and $\pi_{\mu \nu}$ the anisotropic 
stress tensor. The relations that make the identification possible are

$$
\begin{aligned}
\Pi_{\mu \nu} & =G_{\alpha \beta} h^{\alpha}{ }_{\mu} h^{\beta}{ }_{\nu}=p h_{\mu \nu}+\pi_{\mu \nu}, \\
\pi_{\mu \nu} & =\Pi_{\mu \nu}-\frac{1}{3} \Pi^{\kappa}{ }_{\kappa} h_{\mu \nu}=\Pi_{\mu \nu}-p h_{\mu \nu} \\
\rho & =G_{\mu \nu} u^{\mu} u^{\nu}, \quad p=\frac{1}{3} \Pi^{\kappa}{ }_{\kappa} \\
q_{\nu} & =-G_{\alpha \beta} u^{\alpha} h^{\beta}{ }_{n},
\end{aligned}
$$

in which $h_{\mu \nu}$ is the projection tensor orthogonal to velocity $u^{\mu}$ defined by

$h_{\mu \nu}=g_{\mu \nu}+u_{\mu} u_{\nu}, \quad$ with $u_{\mu} u^{\nu}=-1$

In the next section we begin our analysis by selecting the underlying geometry to be that of a Bianchi Type $V$ spacetime; in this case the field equations are reduced to ordinary, coupled differential equations with time as the independent variable.

\section{Solution space}

The general diagonal Bianchi Type V line element is

$$
\begin{aligned}
d s^{2}= & -M(t)^{2} d t^{2}+a(t)^{2} b(t)^{2} d x^{2} \\
& +e^{-2 x}\left(a(t)^{4} d y^{2}+b(t)^{4} d z^{2}\right),
\end{aligned}
$$

with the corresponding isometry generating vector fields:

$\xi_{1}=\frac{\partial}{\partial y}, \quad \xi_{2}=\frac{\partial}{\partial z}, \quad \xi_{3}=\frac{\partial}{\partial x}+y \frac{\partial}{\partial y}+z \frac{\partial}{\partial z}$.

In order to find a compatible initial form for the æther vector field we first note that it should commute with the above generators, which dictates that $u_{\mu}=\left\{\mathrm{u} 0(t), \mathrm{u} 1(t), e^{-x} \mathrm{u} 2(t)\right.$, $\left.e^{-x} \mathrm{u} 3(t)\right\}$. A further restriction is brought about by the assumption that it should be curl-free, i.e. $u_{\mu ; \nu}-u_{\nu ; \mu}=0$. Of course, this extra restriction kills the possibly interesting longitudinal degree of freedom of the æther field, but it also has several advantages: It ensures that in the solutions found $u_{\mu}$ (thus also $u^{\mu}$ ) will be orthogonal to the hyper-surfaces defined by the corresponding potential, thus allowing the recovery of metrics with higher symmetry (e.g. FLRW in an appropriate limit) in which this condition is mandatory; It also makes feasible the possible contact with Horava gravity in which the existence of a physical time $T$ (here identifiable to the aforesaid potential) is of main importance; Last, but not least, it simplifies the (otherwise intractable) equations to be solved. These requirements, along with the use of (2.10), result in the following initial form $\left(\lambda_{1}\right.$ is an integration constant):

$$
\begin{aligned}
u^{a} & =\frac{u_{0}(t)}{-M^{2}} \delta_{t}^{a}+\frac{\lambda_{1}}{a(t)^{2} b(t)^{2}} \delta_{x}^{a}, \\
u_{0}(t) & =\sqrt{a(t)^{2} b(t)^{2}+\lambda_{1}^{2}} .
\end{aligned}
$$

We next make use of the inherent time re-parameterization covariance and select the time gauge so that $M(t)=$ $a(t) b(t)$; thus, the second component of the Eq. (2.9) assumes the form

$$
\begin{gathered}
\lambda_{1} a^{-6} b^{-6}\left(( c _ { 1 } + c _ { 3 } ) \left(-b^{2} a^{2}-a b\left(b a^{\prime \prime}+4 a^{\prime} b^{\prime}\right)\right.\right. \\
\left.\left.+a^{2}\left(-b b^{\prime \prime}-b^{\prime 2}+2 b^{2}\right)\right)+a(t)^{4} b^{4} \lambda\right),
\end{gathered}
$$

implying that we have to separately investigate the two classes of solutions where: Class A when $\lambda_{1}=0$ and Class $\mathrm{B}$ when $\lambda_{1} \neq 0$.

The general approach we adopt in order to reveal the solution space is to algebraically solve two of the equations in terms of the accelerations $a^{\prime \prime}(t), b^{\prime \prime}(t)$ and substitute the result into the rest. In doing so some particular branches appear when the denominators of the corresponding expressions vanish. This may happen either for specific value ranges of the constants or for particular relations among $a(t), b(t)$. In what follows we present all the cases that emerge.

\subsection{Class A solutions}

The strategy for uncovering the solution space is, in a way, similar to that adopted in [36]: one algebraically solves three of the ensuing equations in terms of $\lambda(t), a^{\prime \prime}(t), b^{\prime \prime}(t)$ and substitutes into the rest, thus obtaining a number of equations involving only up to first time derivatives. The consistency of these equations along with the vanishing of the various denominators appearing in the entire process imply that the existence of the solution to the field equations is directly related to the ranges of values of the coefficients $c_{1}, c_{2}, c_{3}$ and $c_{4}$ of the theory. In this Class there are three possible cases of study, corresponding to

$c_{1}+3 c_{2}+c_{3}-2=0$

(Case $\left.A_{1}\right)$

$c_{1}+3 c_{2}+c_{3}-2 \neq 0 \quad q=0$

(Case $\left.A_{2}\right)$

$c_{1}+3 c_{2}+c_{3}-2 \neq 0 \quad q \neq 0$

(Case $\left.A_{3}\right)$

where $q=\frac{1}{5}\left(5 c_{1}+9 c_{2}+5 c_{3}-4\right)$. The only non-zero component of (2.9) fixes the Lagrange multiplier to $\lambda=$ $\frac{1}{5 a(t)^{4} b(t)^{4}}\left(a(t) b(t)\left(2\left(6 c_{2}+5 q+4\right) a^{\prime}(t) b^{\prime}(t)-15 c_{2} a(t)\right.\right.$ $\left.b^{\prime \prime}(t)\right)-5 b(t)^{2}\left(3 c_{2} a(t) a^{\prime \prime}(t)+\left(3 c_{2}-5 q-4\right) a^{\prime}(t)^{2}\right)+$ $\left.5 a(t)^{2}\left(-3 c_{2}+5 q+4\right) b^{\prime}(t)^{2}\right)$ which is then substituted into (2.7), giving the final set of equations to be solved in this class. 


\subsubsection{Case $A_{1}$}

The assumpton leads to $q=\frac{6(1-\mathrm{c} 2)}{5}$ and substituting in (2.7) we calculate the difference $(0,0)-(1,1)=-4$. So there is no solution in this case.

\subsubsection{Case $A_{2}$}

The assumptions of this case make the $(0,0)$ component of (2.7)

$$
\frac{12}{5}\left(1-c_{2}\right) \frac{a^{\prime} b^{\prime}}{a b}-1=0,
$$

indicating that $c_{2} \neq 1$ must hold for solutions to exist. As we can easily see the above equation admits the scaling symmetry $a \rightarrow \omega_{1} a, \quad b \rightarrow \omega_{2} b$; thus, if we make the replacement

$$
\begin{aligned}
& a(t)=\exp \left[\int\left(a_{1}(t)+b_{1}(t)\right) d t\right], \\
& b(t)=\exp \left[\int\left(a_{1}(t)-b_{1}(t)\right) d t\right],
\end{aligned}
$$

the aforementioned equation transforms to

$$
\frac{12}{5}\left(1-c_{2}\right)\left(a_{1}(t)^{2}-b_{1}(t)^{2}\right)-1=0,
$$

which, being a quadratic form in $a_{1}(t), b_{1}(t)$, can be parametrized by

$$
\begin{aligned}
a_{1}(t) & =\frac{m}{2} \sinh (f(t)), b_{1}(t)=\frac{m}{2} \cosh (f(t)), \\
m & =\sqrt{\frac{5}{3\left(c_{2}-1\right)}} .
\end{aligned}
$$

If we substitute the above forms of $a_{1}(t), b_{1}(t)$ into the rest of (2.7) we find that a solution exists if $f(t)$ satisfies the differential equation

$2 \cosh f(t)\left(2 m \cosh f(t)+f^{\prime}(t)\right)=0$,

with solutions

$$
\begin{aligned}
& f(t)=\frac{i \pi}{2}(2 \kappa+1), \kappa \in \mathbb{Z}, \quad \text { or } \\
& f(t)=-2 \tanh ^{-1}\left(\tan \left(m\left(t-t_{0}\right)\right)\right),
\end{aligned}
$$

- If $f(t)=\frac{i \pi}{2}(2 \kappa+1)$, the solution is real if $c_{2}<1$ and then $a(t), b(t)$ becomes

$$
a(t)=b(t)=\exp \frac{\epsilon m}{2} t, \quad \epsilon= \pm 1
$$

yielding the line element

$$
d s^{2}=e^{2 \epsilon m t}\left(-d t^{2}+d x^{2}+e^{-2 x} d y^{2}+e^{-2 x} d z^{2}\right) .
$$

The Riemann tensor is zero when $c_{2}=-\frac{2}{3}$, so we obtain the Minkowski space-time in this case. The corresponding Ricci scalar is

$R=-\frac{2\left(3 c_{2}+2\right)}{c_{2}-1} \exp (-2 \epsilon m t)$

which has a curvature singularity at $t \rightarrow \pm \infty$ depending on the value of $\epsilon$. For this solution the physical components of the energy momentum tensor describe a perfect fluid with energy density and pressure component as follows

$\rho=\frac{3 c_{2}+2}{c_{2}-1} \exp (-2 \epsilon m t), p=-\frac{1}{3} \rho$.

- If $f(t)=-2 \tanh ^{-1}\left(\tan \left(m\left(t-t_{0}\right)\right)\right)$, the solution of the field equations is given by the functions

$$
\begin{aligned}
& a(t)=\sqrt{\sin \left(m\left(t-t_{0}\right)\right)+\cos \left(m\left(t-t_{0}\right)\right)}, \\
& b(t)=\sqrt{\sin \left(m\left(t-t_{0}\right)\right)-\cos \left(m\left(t-t_{0}\right)\right)},
\end{aligned}
$$

Hence, the line element reads

$$
\begin{aligned}
d s^{2}= & \cos \left(2 m\left(t-t_{0}\right)\right)\left(d t^{2}-d x^{2}\right) \\
& +e^{-2 x}\left(1+\sin \left(2 m\left(t-t_{0}\right)\right)\right) d y^{2} \\
& -e^{-2 x}\left(1-\sin \left(2 m\left(t-t_{0}\right)\right)\right) d z^{2}
\end{aligned}
$$

with $c_{2}>1$. The corresponding Ricci scalar is $\left(3\left(m^{2}+1\right) \cos (4 m(t-\right.$ to $\left.))+7 m^{2}+3\right)$

$\sec ^{3}(2 m(t-$ to $))$ which develops curvature singularities at finite intervals.

It is interesting to note that as the argument $\tau \equiv$ $2 m\left(t-t_{0}\right)$ spans the prime interval $[0,2 \pi]$ the role of the coordinates $t, x$ as time-like and space-like respectevely is interchanged (i.e. when $\frac{\pi}{2}<\tau<\frac{3 \pi}{2} t$ is time-like while when $\frac{3 \pi}{2}<\tau<\frac{\pi}{2} x$ is time-like). The $g_{y y}$ component is always positive (or zero for $\tau=0,2 \pi$ ) with the $g_{z z}$ component exhibiting the opposite behaviour. Thus, in the entire prime interval of $\tau$, the signature of the line element is neutral.

The norms $\left|\xi_{i}\right|^{2} \equiv g_{\mu \nu} \xi_{i}^{\mu} \xi_{i}^{\nu}$ of the Killing vectors are $\left|\xi_{1}\right|^{2}=e^{-2 x}(\sin (\tau)+1),\left|\xi_{2}\right|^{2}=e^{-2 x}(\sin (\tau)-1)$, $\left|\xi_{3}\right|^{2}=e^{-2 x}\left(-e^{2 x} \cos (\tau)+\sin (\tau)\left(y^{2}+z^{2}\right)+y^{2}-z^{2}\right)$. The first two vanish only at the endpoints of the prime interval $\tau=\frac{3 \pi}{2}, \frac{\pi}{2}$ respectively, while the third becomes zero inside the interval of interest. All these can be suggestive that horizons or domain walls exist; However, the neutral character of the signature somewhat obscures the issue.

The above line element corresponds to a perfect fluid 
solution with

$$
\begin{aligned}
& \rho=\frac{3-m^{2}+3\left(1+m^{2}\right) \cos \left(4 m\left(t-t_{0}\right)\right)}{2 \cos ^{3}\left(m\left(t-t_{0}\right)\right)} \\
& p=-\frac{1+5 m^{2}+\left(1+m^{2}\right) \cos \left(4 m\left(t-t_{0}\right)\right)}{2 \cos ^{3}\left(m\left(t-t_{0}\right)\right)}
\end{aligned}
$$

with equation of state parameter

$$
w=-\frac{1+5 m^{2}+\left(1+m^{2}\right) \cos \left(4 m\left(t-t_{0}\right)\right)}{3-m^{2}+3\left(1+m^{2}\right) \cos \left(4 m\left(t-t_{0}\right)\right)}
$$

\subsubsection{Case $\mathrm{A}_{3}$}

In this case we first solve the $(2,2)$ and $(3,3)$ components of (2.7) in terms of the accelerations $a^{\prime \prime}(t), b^{\prime \prime}(t)$. The denominator has the term $6\left(c_{2}-1\right)+5 q$, so we have to check what happens if $q=\frac{6}{5}\left(1-c_{2}\right)$; in this case from the components $(0,0)$ and $(1,1)$ of $(2.7)$ we have $(0,0)-(1,1)=-4$, thus we run into incompatibility.

With $q \neq \frac{6}{5}\left(1-c_{2}\right)$ we substitute the above mentioned accelerations into the equation $(2.7)$. The $(0,0)$ component of the latter equation admits a scaling symmetry $a \rightarrow$ $\omega_{1} a, \quad b \rightarrow \omega_{2} b$; thus, if we make a replacement similar to (3.6), namely $a(t)=\exp \left[\int\left(-a_{1}(t)+b_{1}(t)\right) d t\right], \quad b(t)=$ $\exp \left[\int\left(a_{1}(t)+b_{1}(t)\right) d t\right]$, the $(0,0)$ component of the field equations is simplified as

$$
\begin{aligned}
& \frac{1}{30} a_{1}(t)^{2}\left(8\left(9 c_{2}-5 q-9\right)\right) \\
& \quad-\frac{1}{30} b_{1}(t)^{2}\left(12\left(6 c_{2}+5 q-6\right)\right)=1 .
\end{aligned}
$$

In order to parametrize the above equation, we have to check the case $q=\frac{1}{5}\left(9 c_{2}-9\right)$. Note that the alternative $\left(6 c_{2}+5 q-\right.$ 6) $=0$ implies $q=\frac{6}{5}\left(1-c_{2}\right)$ which leads to incompatibility as mentioned in the beginning of this section.

- If $q=\frac{9}{5}\left(c_{2}-1\right)$ then the Eq. (3.20) gives

$$
b_{1}(t)= \pm\left(\sqrt{6} \sqrt{1-c_{2}}\right)^{-1}
$$

Then we substitute the above form of $a(t), b(t)$ into the equations of the accelerations and we arrive at a differential equation for $a_{1}(t)$

$a_{1}^{\prime}(t) \pm 4\left(\sqrt{6} \sqrt{1-c_{2}}\right)^{-1} a_{1}(t)=0$

which has the solution $a_{1}(t)=m_{1} \exp (\mp 4 n t)$ with $m_{1}$ an integration constant and $n^{-1}=\sqrt{6\left(1-c_{2}\right)}$.
Finally the scale factors $a(t), b(t)$ are given by

$a(t)=\exp \epsilon\left(2 n t+c e^{-4 n t}\right)$,
$b(t)=\exp \epsilon\left(2 n t-c e^{-4 n t}\right)$,

where $\epsilon^{2}=1, c$ is a redefinition of $m_{1}$ and $c 2<1$. The corresponding Ricci scalar reads

$R=2 e^{-4 n t(\epsilon+2)}\left(64 c^{2} n^{2}-3 e^{8 n t}\left(1-4 n^{2}\right)\right)$,

which has curvature singularities at $t \rightarrow \pm \infty$ for $\epsilon=-1$ and at $t \rightarrow-\infty$ for $\epsilon=1$.

The above solution describes a perfect fluid when $\epsilon=1$, and a non perfect one for $\epsilon=-1$.

The energy density and the pressure component are given by

$$
\begin{aligned}
& \rho=e^{-4 n(\epsilon+2) t}\left(3 e^{8 n t}\left(4 n^{2}-1\right)-64 c^{2} n^{2}\right), \\
& p=-e^{-4 n(\epsilon+2) t}\left(e^{8 n t}\left(4 n^{2}-1\right)+64 c^{2} n^{2}\right),
\end{aligned}
$$

while the nonzero components of the stress tensor $\pi_{\mu \nu}$ are

$$
\begin{aligned}
\pi_{y y}= & -\pi_{z z}=-32 c n^{2}(\epsilon-1) \\
& \exp \left(-4 n t-2 x+4 c \epsilon e^{-4 n t} \sqrt{2 n^{2}+1}\right)
\end{aligned}
$$

- If $\left(9 c_{2}-5 q-9\right) \neq 0$ Eq. (3.20) can be parametrized as

$$
\begin{aligned}
& a_{1}(t)=\frac{\sqrt{15} \cosh (f(t))}{2 \sqrt{\left(9 c_{2}-5 q-9\right)}}, \\
& b_{1}(t)=\frac{\sqrt{5} \sinh (f(t))}{\sqrt{2\left(6 c_{2}+5 q-6\right)}} .
\end{aligned}
$$

Now, if we substitute the above values of $a_{1}(t), b_{1}(t)$ into the rest of (2.7) we find that a solution exists if $f(t)$ satisfies the differential equation

$$
\begin{aligned}
& \cosh (f(t))\left(\sqrt{10}\left(6 c_{2}+5 q-6\right) f^{\prime}(t)\right. \\
& \left.+20 \sqrt{6 c_{2}+5 q-6} \cosh (f(t))\right)=0 .
\end{aligned}
$$

- When $\cosh (f(t))=0$ we have

$$
f(t)=\frac{i \pi}{2}(2 \kappa+1), \kappa \in \mathbb{Z}
$$


For these functions $f(t)$, the solution of the field equations is determined to be

$$
a(t)=b(t)=e^{\epsilon k t}, \quad k=\sqrt{\frac{5}{2\left(6-5 q-6 c_{2}\right)}},
$$

and the line element becomes

$$
d s^{2}=e^{4 \epsilon k t}\left(-d t^{2}+d x^{2}+e^{-2 x} d y^{2}+e^{-2 x} d z^{2}\right) .
$$

In the special case when $q=-\frac{1}{5}\left(6 c_{2}+4\right)$ the Riemann tensor is 0 , and we thus obtain the Minkowski spacetime.

For any other value of $q$ the corresponding Ricci scalar is

$$
R=-6 e^{-4 \epsilon k t}\left(4 k^{2}-1\right),
$$

which has a curvature singularity at $t \rightarrow \pm \infty$ depending on $\epsilon$, being plus or minus 1 . The energy momentum tensor describes a perfect fluid, similarly to the previous case.

- When $\cosh f(t) \neq 0$ the solution of the differential Eq. (3.28) is

$$
\begin{aligned}
f(t) & =-2 \tanh ^{-1}\left(\tan \left(A\left(t-t_{0}\right)\right)\right), \\
A & =\sqrt{10\left(6 c_{2}+5 q-6\right)^{-1}},
\end{aligned}
$$

which implies that,

$$
\begin{aligned}
& a(t)=\cos ^{1 / 4} 2 A\left(t-t_{0}\right)\left(\frac{1+\tan A\left(t-t_{0}\right)}{1-\tan A\left(t-t_{0}\right)}\right)^{-B}, \\
& b(t)=\cos ^{1 / 4} 2 A\left(t-t_{0}\right)\left(\frac{1+\tan A\left(t-t_{0}\right)}{1-\tan A\left(t-t_{0}\right)}\right)^{B}
\end{aligned}
$$

with $B=\sqrt{3\left(8\left(6-5 A^{2} q\right)\right)^{-1}}$.

The corresponding line element is

$$
\begin{gathered}
d s^{2}=\cos (2 A t)\left(-d t^{2}+d x^{2}+e^{-2 x}\left(\left(\frac{1+\tan A t}{1-\tan A t}\right)^{-4 B}\right.\right. \\
\left.\left.d y^{2}+\left(\frac{1+\tan A t}{1-\tan A t}\right)^{4 B} d z^{2}\right)\right)
\end{gathered}
$$

where we removed $t_{0}$ by a redefinition of the coordinate $t \mapsto t+t_{0}$. It is easy to see that this line element describes a perfect fluid solution.

The Ricci scalar is given by

$$
\begin{aligned}
R= & -\left(3+A^{2}\left(9-32 B^{2}\right)\right. \\
& \left.+3\left(A^{2}+1\right) \cos (4 A t)\right) \sec ^{3}(2 A t),
\end{aligned}
$$

which diverges for $t=\frac{\pi}{4 A}(2 \kappa+1), \kappa \in \mathbb{Z}$.

We proceed our analysis with the derivation of the secondclass of solutions in which $u^{a}$ is not the comoving observer.

\subsection{Class B solutions}

For the second class of solutions where $\lambda_{1} \neq 0$, the strategy is now to first solve the second component of (2.9) for the Lagrange multiplier $\lambda(t)$ obtaining:

$$
\begin{aligned}
\lambda(t)= & -\left(c_{1}+c_{3}\right)\left(-a b^{2} a^{\prime \prime}-4 a b a^{\prime} b^{\prime 2} a^{\prime 2}\right. \\
& \left.-a^{2} b b^{\prime \prime 2} b^{\prime 2}+2 a^{2} b^{2}\right)(a b)^{-4} .
\end{aligned}
$$

Next we substitute this $\lambda(t)$ into the first component of (2.9) and also into the components of (2.7).

If, from the resulting equations, we solve the first component of (2.9) and the (2,2) component of (2.7) in terms of the accelerations $a^{\prime \prime}(t), b^{\prime \prime}(t)$, the possible vanishing of the following expression

$$
\begin{aligned}
& \left(a^{2} b^{2}\left(c_{1}+c_{3}+1\right)+\lambda_{1}^{2}\left(c_{1}+c_{3}\right)\right) \\
& \quad\left(a(t)^{2} b(t)^{2}\left(c_{1}+3 c_{2}+c_{3}\right)+2 c_{2} \lambda_{1}^{2}\right)
\end{aligned}
$$

appearing in the denominators of $a^{\prime \prime}(t), b^{\prime \prime}(t)$ must be considered, giving rise to the following cases

$$
a^{2} b^{2}\left(c_{1}+c_{3}+1\right)+\lambda_{1}^{2}\left(c_{1}+c_{3}\right)=0 \quad c_{1}+c_{3}+1 \neq 0
$$

(Case $B_{1}$ )

$c_{2}=0 \quad c_{1}+c_{3}=0$

(Case $\left.B_{2}\right)$

$a^{2} b^{2}\left(c_{1}+3 c_{2}+c_{3}\right)+2 c_{2} \lambda_{1}^{2}$

(Case $B_{3}$ )

$=0 \quad c_{1}+3 c_{2}+c_{3} \neq 0, q \neq 0$

$a^{2} b^{2}\left(c_{1}+3 c_{2}+c_{3}\right)+2 c_{2} \lambda_{1}^{2}=0 \quad c_{1}+c_{3} \neq 0 \quad\left(\right.$ Case $\left.B_{4}\right)$

\subsubsection{Case $B_{1}$}

This case finally results into $a, b$ being both constant functions. When we substitute these constant values in (2.7), we end up with $(0,0)+(1,1)=-2\left(c_{1}+c_{3}+1\right)=0$ which is impossible; thus there is no solution in this case.

\subsubsection{Case $\mathrm{B}_{2}$}

With the above assumption the constrain equation, i.e. the $(0,0)$ component of $(2.7)$, becomes

$\frac{8 a^{\prime} b^{\prime}}{a b}+\frac{2 a^{\prime 2}}{a^{2}}+\frac{2 b^{\prime 2}}{b^{2}}-3=0$. 
As before, the existing scaling symmetry indicates that the replacement

$$
\begin{aligned}
& a(t)=\exp \left[\frac{1}{2} \int(\sqrt{3} \sinh (f(t))+\cosh (f(t))) d t\right] \\
& b(t)=\exp \left[\frac{1}{2} \int(\cosh (f(t))-\sqrt{3} \sinh (f(t))) d t\right]
\end{aligned}
$$

satisfies the above expression, while the rest of the equations (2.7) are solved if $f(t)$ obeys the first order differential equation

$$
-2 \sinh (f(t))\left(f^{\prime}(t)+2 \sinh (f(t))\right)=0 .
$$

Its solutions read

$$
f(t)=i \kappa \pi, \kappa \in \mathbb{Z}, \quad \text { or } \quad f(t)=2 \operatorname{coth}^{-1} e^{2 t-m_{1}},
$$

where $m_{1}$ is an integration constant.

- If $f(t)=i \kappa \pi$ the solution is

$$
a(t)=e^{\frac{t \epsilon}{2}}, \quad b(t)=e^{\frac{t \epsilon}{2}},
$$

and the line element becomes

$$
d s^{2}=e^{2 \epsilon t}\left(-d t^{2}+d x^{2}+e^{-2 x} d y^{2}+e^{-2 x} d z^{2}\right)
$$

with the Riemann tensor equal to zero, indicating the Minkowski space time.

- If $f(t)=2 \operatorname{coth}^{-1} e^{2 t-m_{1}}$ the solution is:

$$
\begin{aligned}
& a(t)=e^{-\frac{t}{2}}\left(\frac{e^{m_{1}}-e^{2 t}}{e^{m_{1}}+e^{2 t}}\right)^{\frac{\sqrt{3}}{4}} \sqrt[4]{1-e^{4 t-2 m_{1}}} \\
& b(t)=e^{-\frac{t}{2}}\left(\frac{e^{m_{1}}-e^{2 t}}{e^{m_{1}}+e^{2 t}}\right)^{-\frac{\sqrt{3}}{4}} \sqrt[4]{1-e^{4 t-2 m_{1}}}
\end{aligned}
$$

with the corresponding line-element

$$
\begin{aligned}
d s^{2}= & \kappa^{2} \sinh (2 \tau)\left(d \tau^{2}-d x^{2}+e^{-2 x} \tanh ^{\sqrt{3}}(\tau) d y^{2}\right. \\
& \left.+e^{-2 x} \tanh ^{-\sqrt{3}}(\tau) d z^{2}\right)
\end{aligned}
$$

where $\kappa^{2}=2 e^{-m 1}$ and $\tau=t+\ln \left(\frac{\kappa}{\sqrt{2}}\right)$.

The Ricci tensor is derived to be $R_{\mu \nu}=0$; thus the above line element,while being a vacuum of the present theory, also describes a GR vacuum solution. The Kretsmann scalar equals

$$
K r \equiv R^{\mu \nu \kappa \lambda} R_{\mu \nu \kappa \lambda}=\frac{96}{\kappa^{4}} \operatorname{csch}^{6}(2 \tau),
$$

signaling the existence of a singularity at $\tau=0$. Note that $\kappa$ is a gravitational essential constant and that the time-like character between $\tau, x$ is interchanged as $\tau$ spans the interval $(-\infty, \infty)$. However the components $g_{y y}, g_{z z}$ of the metric described by the above line element become imaginary for $\tau \in(-\infty, 0)$; consequenly, the allowed range is $\tau \in(0, \infty)$ with the singularity residing at $\tau=0$. In this range the signature is $(+,-,+,+)$ indicating that $x$ is the time-like variable. The norms $\left|\xi_{i}\right|^{2} \equiv g_{\mu \nu} \xi_{i}^{\mu} \xi_{i}^{\nu}$ of the Killing vectors are $\left|\xi_{1}\right|^{2}=k^{2} e^{-2 x} \sinh (2 \tau) \tanh ^{\sqrt{3}}(\tau),\left|\xi_{2}\right|^{2}=$ $k^{2} e^{-2 x} \sinh (2 \tau) \tanh ^{-\sqrt{3}}(\tau), \quad\left|\xi_{3}\right|^{2}=k^{2} \sinh (2 t)$ $\left(e^{-2 x} y^{2} \tanh ^{\sqrt{3}}(\tau)+e^{-2 x} z^{2} \tanh ^{-\sqrt{3}}(\tau)-1\right)$. The first two are positively defined in the allowed range for $\tau$ and $x \in(-\infty,+\infty)$, but the third vanishes on the hypersurface $e^{2 x}=y^{2} \tanh ^{\sqrt{3}}(\tau)+z^{2} \tanh ^{-\sqrt{3}}(\tau)$ indicating a complicated partition of the spacetime in causally disconnected regions. Of course, a more detailed description requires the investigation of the geodesic structure which lies well outside the scope of the present work.

\subsubsection{Case $B_{3}$}

In this case we end up with $a(t) b(t)=\lambda_{1} \sqrt{-2 c_{2}}\left(c_{1}+3 c_{2}+\right.$ $\left.c_{3}\right)^{-1 / 2}$ and if we substitute this expression into the components of (2.7) we can see that $a(t)$ must be constant, which entails the result $(0,0)+(1,1)=-2\left(c_{1}+c_{3}+1\right)=0$. However, this relationship makes the rest of Eq. (2.7) incompatible with the assumptions of the given branch; we thus conclude that there is no solution.

The above analysis corresponds to the last case where the denominators vanish. Thus we can now use the forms of the accelerations $a^{\prime \prime}(t), b^{\prime \prime}(t)$ and replace them into Eq. (2.7). This action results in the $(0,1)$ component being

$$
\begin{aligned}
& 2 \lambda_{1}\left(c_{1}+c_{3}\right)\left(\lambda_{1} b a^{\prime}\right. \\
& \left.+a\left(b \sqrt{a^{2} b^{2}+\lambda_{1}^{2}}+\lambda_{1} b^{\prime}\right)\right)(a b)^{-3}=0 .
\end{aligned}
$$

Various cases will emerge from the above equation. When $c_{1}+c_{3}=0$, the only independent components of (2.7) are the $(0,0)$ and $(1,1)$ which are quadratic in the velocities. If we differentiate these with respect to $t$, use again the accelerations and subtract appropriate linear combinations of the expressions themselves, we end up with the following two cases:

- If $c_{2} \neq 0, c_{1}+c_{3}=0, a b=c_{b}$ then the combination $(0,0)-(1,1)$ of the components of $(2.7)$ reads $-\frac{4 \mathrm{c} 2 \lambda_{1}^{2}}{\mathrm{cb}^{2}}-4=0$ implying $\mathrm{cb}=i \sqrt{\mathrm{c} 2} \lambda_{1}$ and thus a neutral signature. With this value for $\mathrm{cb}$ the $(0,0)$ component of (2.7) becomes 


$$
-\frac{4 a^{\prime}(t)^{2}}{a(t)^{2}}-1=0,
$$

which has the solution

$$
a(t)=e^{m_{1}+\frac{i t \epsilon}{2}}
$$

with $\epsilon= \pm 1, m_{1}$ an integration constant, and all equations have now been sattisfied.

At this stage the redefinitions $\left\{y \rightarrow \mu e^{-2 m_{1}} Y, z \rightarrow\right.$ $\left.\frac{e^{2 m_{1}} Z}{\mu}, t \rightarrow i T, x \rightarrow X, \mathrm{c} 2 \rightarrow \frac{\mu^{2}}{\lambda_{1}^{2}}\right\}$ cast the line element into the simple final form

$$
\begin{aligned}
d s^{2}= & \mu^{2}\left(-d T^{2}-d X^{2}+e^{-2 T \epsilon-2 X} d Y^{2}\right. \\
& \left.+e^{2 T \epsilon-2 X} d Z^{2}\right)
\end{aligned}
$$

while the aether one-form transform into $u_{\mu}=\left\{-\sqrt{\mu^{2}-\lambda_{1}^{2}}, \lambda_{1}, 0,0\right\}$ which, in order to be real, implies the restriction $\lambda 1^{2}<|\mu|^{2}$.

The above line-element represents a singularity free geometry, on account of having vanishing covariant derivative of its Riemmann tensor and all fourteen curvature scalars monomialls of the Ricci scalar $R=\frac{8}{\mu^{2}}$. Note that, since the space admits only one extra Killing field $\xi_{4}=(1,0, \epsilon Y,-\epsilon Z)$, we have the intersting case of a CSI space time [44]

Despite the non physical character of a neutral singnature line element, it is not unreasonable to investigate the resulting effecting $T_{\mu \nu}$ in terms of perfect fluid:

$$
\rho=\frac{2}{\mu^{2}}, p=-\frac{2}{\mu^{2}}, q_{\kappa}=0, \pi_{\mu \nu}=0
$$

inferring that it corresponds to an isotropic perfect fluid with an equation of state $p+\rho=0$ and zero heat conduction, i.e. it mimics that of a cosmological constant.

- If $c_{2} \neq 0, c_{1}+c_{3}=0, a b \neq c_{b}$ then with the application of the same steps as before we end up with $a b=$ const., which contradicts the initial assumption of this case. Thus there is no solution here although, of course, the previous solution holds true.

\subsubsection{Case $\mathrm{B}_{4}$}

Now we can solve Eq. (3.47) as a differential equation for $b(t)$ obtaining

$b(t)=\frac{2 \lambda_{1}^{2} e^{m_{1}+t}}{e^{2 t} a(t)-\lambda_{1}^{2} e^{2 m_{1}} a(t)}$,

with $m_{1}$ an integration constant.
When this is substituted into (2.7) we get a differential equation for $a(t)$ which leads to the final scale factors

$$
\begin{aligned}
& a(t)=\frac{m_{2} e^{t / 2}}{\sqrt{e^{2 t}-\lambda_{1}^{2} e^{2 m_{1}}}}, \\
& b(t)=\frac{2 \lambda_{1}^{2} e^{m_{1}+\frac{t}{2}}}{m_{2} \sqrt{e^{2 t}-\lambda_{1}^{2} e^{2 m_{1}}}}
\end{aligned}
$$

under the assumpion $c_{1}+3 c_{2}+c_{3}=2$.

The resulting line element is

$d s^{2}=\lambda_{1}^{2} \operatorname{csch}^{2} t\left(-d t^{2}+d x^{2}+e^{-2 x} d y^{2}+e^{-2 x} d z^{2}\right)$,

where we have used the transformation $t \mapsto t+m_{1}+$ $\ln \lambda_{1}, x \mapsto x+m_{1}^{2}+\ln 2, y \mapsto 4 m_{2}^{-2} \lambda_{1}^{2} y, z \mapsto$ $e^{-2 m_{1}} \lambda_{1}^{-2} m_{2}^{2} z$.

Furthermore, it holds that $R_{\lambda \mu \nu \kappa}=\frac{1}{\lambda_{1}^{2}}\left(g_{\lambda \nu} g_{\mu \kappa}-g_{\lambda \kappa} g_{\mu \nu}\right)$, revealing the space-time as maximally symmetric with Ricci scalar $R=\frac{12}{\lambda_{1}^{2}}$.. If we further investigate the physical properties of the energy-momentum tensor for the above solution, we find that

$\rho=\frac{3}{\lambda_{1}^{2}}, p=-\frac{3}{\lambda_{1}^{2}}, q_{\kappa}=0, \pi_{\mu \nu}=0$

inferring that it corresponds to an isotropic perfect fluid with an equation of state $p+\rho=0$ and zero heat conduction, i.e. it mimics that of a cosmological constant.

\section{Conclusion}

In this work we studied the existence of exact solutions for the field equations in the Lorentz violating theory known as Einstein-aether gravity. For the background geometry we assumed that of a Bianchi Type $\mathrm{V}$ spacetime, with a diagonal scale factor matrix dictated by the vanishing of the $G_{t x}$ constraint equation. Note that this form of the lineelement, despite having two independent functions of time, is not Locally Rotationally Symmetric, i.e. it does not admit a fourth Killing field. We have also restricted the form of the aether field by imposing on it the symmetries of the geometry, thus arriving at two possible cases, that of tilted or nontilted $u_{\mu}$. For both models of our study we determined the field equations and exhibited their solution space. Like in the previous similar work of ours concerning Bianchi Type III geometry [36], we find here also that exact solutions exist only for specific values of the coupling parameters of the Einstein-aether theory.

When the aether field is parallel to the comoving observer, we found that there exists an exact isotropic solution in case 
Table 1 Physically acceptable exact Bianchi V spacetimes in Einstein-aether theory

\begin{tabular}{llll}
\hline Case & Condition & Isotropic spacetime & Ricciscalar $R$ \\
\hline$A_{3 i}$ & $\left(9 c_{2}-5 q-9\right) \neq 0, f(t)=\frac{i \pi}{2}(2 \kappa+1)$ & Yes & $-6 e^{-4 \epsilon k t}\left(4 k^{2}-1\right)$ \\
$A_{3 i i}$ & $\left(9 c_{2}-5 q-9\right) \neq 0, f(t)=-2 \tanh ^{-1}\left(\tan \left(A\left(t-t_{0}\right)\right)\right)$ & No & $-\left(\rho_{0}+\rho_{1} \cos \left(4 \rho_{2} t\right)\right) \sec ^{3}\left(2 \rho_{2} t\right)$ \\
$B_{2 i}$ & $c_{2}=0, f(t)=i \kappa \pi$ & Yes (Minkowski) & 0 \\
$B_{2 i i}$ & $c_{2}=0, f(t)=2 \operatorname{coth}^{-1} e^{2 t-m_{1}}$ & No & $\left.\frac{96}{\kappa^{4}} \operatorname{csch}(2 \tau)\right)$ \\
$B_{3}$ & $c_{1}+c_{3}=0, a(t) b(t)=c_{b}$ & No & $\frac{8}{\mu^{2}}($ singularity free) \\
$B_{4}$ & $c_{1}+3 c_{2}+c_{3}=2$ & Yes & $\frac{12}{\lambda_{1}^{2}}$ (singularity free) \\
\hline
\end{tabular}

$A_{2}$, when $c_{1}+3 c_{2}+c_{3}-2 \neq 0, q=0$. In this case, the spacetime reduces to the Friedmann-Lemaître-RobertsonWalker spacetime with non-zero spatial curvature. On the other hand, in case $A_{3}$, when $c_{1}+3 c_{2}+c_{3}-2 \neq 0, q \neq 0$ both anisotopic and isotropic solutions are found; the latter case being when $\left(9 c_{2}-5 q-9\right) \neq 0$, an isotropic solution is found.

In a similar way when the aether field is not parallel to the comoving observer anisotropic and isotropic exact solutions are found for specific relations between the coupling constants of the Einstein-aether theory. There are two possible constraining relations for the coupling constants, when exact solutions exist. These occur in case $B_{2}$ where $c_{2}=0, c_{1}+c_{3}=0$, and in case $B_{6}$ where $c_{1}+c_{3} \neq 0$.

Lastly, we would like to briefly comment on the LRS case: The scale factor matrix is now $\gamma_{\mu \nu}=\operatorname{diag}\left\{a^{2}, a^{2}, b^{2}\right\}$ which results in a non-zero $G_{t x}$ component of the Einstein tensor. Now the geometry admits the fourth Killing field $\xi_{4}=-z \frac{\partial}{\partial y}+y \frac{\partial}{\partial z}$. The aether field remains the same, since it commutes with $\xi_{4}$, and thus the classification according to $\lambda_{1}$ being zero or non-zero is also valid. There is however a substantial difference in the solution space. For example in the class A case $\left(\lambda_{1}=0\right)$ there is only one family of solutions described by the scale factors $a(t)=m_{2} \exp \frac{(\sqrt{2} t) \epsilon}{m_{1} \sqrt{(2-Q)}}$ and $b(t)=m_{1} a(t)$ where $\epsilon= \pm 1, Q=c_{1}+3 c_{2}+c 3<2$ for the solution to be real, with the Minkowski space-time corresponding to the value $Q=0$. Note also that, if a free electromagnetic field was considered instead of the aether field, the line element used in the present work would lead only to the vacuum solution whereas the LRS element would have non-trivial solutions. All these issues are under consideration and will be presented in a future publication.

Before we end our discussion, a brief word is pertinent, concerning the viability of the exact solutions here found when they are examined in the light of the values and/or value ranges of the aether coupling constants given by observations (see, e.g. [45-47]). In [45] the gravitational wave data of the event GW170817 have been applied, while in [46] the constraints have been produced by using ultra-high energy cosmic rays.
With the use of the results of these works, we conclude that the solution of case $A_{2}$ is not viable since $c_{2}$ is not in the range of values as given by [45]. For the case $A_{3}$, only the solution with $\left(9 c_{2}-5 q-9\right) \neq 0$ is physicaliy acceptable, while the exact solution in which $q=\frac{9}{5}\left(c_{2}-1\right)$ is rejected by the observations.

As for the family $\mathrm{B}$ of solutions, $B_{2}$ and $B_{3}$ are acceptaple solutions if they are seen in their lower limits $c_{2} \rightarrow 0$ and $c_{1}+c_{3} \rightarrow 0$ respectively. Finally, for case $B_{4}$, only the de Sitter solution does not satisfy the constraints. The results are summarized in the Table 1.

Data Availability Statement This manuscript has associated data in a data repository. [Authors' comment: This manuscript has associated data in arXiv:1911.03660 [gr-qc].]

Open Access This article is licensed under a Creative Commons Attribution 4.0 International License, which permits use, sharing, adaptation, distribution and reproduction in any medium or format, as long as you give appropriate credit to the original author(s) and the source, provide a link to the Creative Commons licence, and indicate if changes were made. The images or other third party material in this article are included in the article's Creative Commons licence, unless indicated otherwise in a credit line to the material. If material is not included in the article's Creative Commons licence and your intended use is not permitted by statutory regulation or exceeds the permitted use, you will need to obtain permission directly from the copyright holder. To view a copy of this licence, visit http://creativecomm ons.org/licenses/by/4.0/.

Funded by SCOAP ${ }^{3}$.

\section{References}

1. C.W. Misner, Astrophys. J. 151, 431 (1968)

2. K.C. Jacobs, Astrophys. J. 153, 661 (1968)

3. C.B. Collins, S.W. Hawking, Astrophys. J. 180, 317 (1973)

4. J.D. Barrow, Mon. Not. R. Astron. Soc. 175, 359 (1976)

5. MPJr Rayan, L.C. Shepley, Homogeneous Relativistic Cosmologies (Princeton University Press, Princeton, 1975)

6. P.A. Terzis, (2013). arXiv:1304.7894 [math.RT]

7. E. Kasner, Am. J. Math. 43, 217 (1921)

8. D. Lorenz, Phys. Lett. A 79, 19 (1980)

9. J. Hajj-Boutros, J. Math. Phys. 27, 1592 (1986)

10. T. Christodoulakis, P.A. Terzis, J. Math. Phys. 47, 102502 (2006)

11. T. Christodoulakis, P.A. Terzis, Class. Quant. Gravit. 24, 875 (2007)

12. A. Harvey, D. Tsoubelis, Phys. Rev. D 15, 2734 (1977) 
13. P.A. Terzis, T. Christodoulakis, Class. Quant. Gravit. 29, 235007 (2012)

14. D. Lorenz, Astrophys. Space. Sci. 85, 69 (1982)

15. P.A. Terzis, T. Christodoulakis, Gen. Relat. Gravit. 41, 469 (2009)

16. D. Lorenz, Phys. Rev. D 22, 1848 (1980)

17. N. Dimakis, P.A. Terzis, T. Christodoulakis, Phys. Rev. D 99, 023536 (2019)

18. A.P. Billyard, A.A. Coley, R.J. van den Hoogen, J. Ibanez, I. Olagasti, Class. Quant. Gravit. 16, 4035 (1999)

19. J.M. Aguirregabiria, A. Feinstein, J. Ibanez, Phys. Rev. D 48, 4662 (1993)

20. M. Tsamparlis, A. Paliathanasis, Gen. Relat. Gravit. 43, 1861 (2011)

21. A. Banerjee, N.O. Santos, Il Nuovo Cimento B 67, 31 (1982)

22. B.K. Nayak, G.B. Bhuyan, Gen. Relat. Gravit. 19, 939 (1987)

23. R. Venkateswarlu, J. Satish, Int. J. Theor. Phys. 53, 1879 (2014)

24. A. Paliathanasis, L. Karpathopoulos, A. Wojnar, S. Capozziello, EPJC 76, 225 (2016)

25. A. Paliathanasis, J.D. Barrow, P.G.L. Leach, Phys. Rev. D 94, 023525 (2016)

26. A. Paliathanasis, J.L. Said, J.D. Barrow, Phys. Rev. D 97, 044008 (2018)

27. T. Pailas, P.A. Terzis, T. Christodoulakis, Class. Quant. Gravit. 35, 145003 (2018)

28. W. Donnelly, T. Jacobson, Phys. Rev. D 82, 064032 (2010)

29. W. Donnelly, T. Jacobson, Phys. Rev. D 82, 081501 (2010)
30. C. Heinicke, P. Baekler, F.W. Hehl, Phys. Rev. D 72, 025012 (2005)

31. H. Wei, X.-P. Yan, Y.-N. Zhou, Gen. Relat. Gravit. 46, 1719 (2014)

32. X. Meng, X. Du, Phys. Lett. B 710, 493 (2012)

33. J.D. Barrow, Phys. Rev. D 85, 047503 (2012)

34. C. Armendariz-Picon, N.F. Sierr, J. Garriga, JCAP 1007, 010 (2010)

35. R.A. Battye, F. Pace, D. Trinh, Phys. Rev. D 96, 064041 (2017)

36. M. Roumeliotis, A. Paliathanasis, P.A. Terzis, T. Christodoulakis, EPJC 79, 349 (2019)

37. A. Paliathanasis, Inhomogeneous spacetimes in Einstein-æther Cosmology, submitted (2019)

38. A.A. Coley, G. Leon, P. Sandin, J. Latta, JCAP 15, 12 (2015)

39. J. Latta, G. Leon, A. Paliathanasis, JCAP 16, 051 (2016)

40. B. Alhulaimi, R.J. van den Hoogen, A.A. Coley, JCAP 17, 045 (2017)

41. A.A. Coley, G. Leon, P. Sandin, J. Latta, JCAP 12, 010 (2015)

42. A. Coley, G. Leon, Static Spherically Symmetric Einstein-aether Models. arXiv: 1905.02003

43. T. Jacobson, Phys. Rev. D 89, 081501 (2014)

44. A. Coley, S. Hervik, N. Pelavas, Class. Quant. Gravit. 23, 3053 (2006)

45. J. Oost, S. Mukohyama, A. Wang, Phys. Rev. D 97, 124023 (2018)

46. J.W. Elliott, G.D. Moore, H. Stoica, JHEP 0508, 066 (2005)

47. K. Yagi, D. Blas, N. Yunes, E. Barausse, Phys. Rev. Lett. 112(16), $161101(2014)$ 